\title{
Terahertz spectroscopy of methanimine and its isotopologs ${ }^{\star}$
}

\author{
Yuta Motoki $^{1}$, Fuya Isobe ${ }^{1}$, Hiroyuki Ozeki ${ }^{1}$, and Kaori Kobayashi ${ }^{2}$ \\ ${ }^{1}$ Department of Environmental Science, Faculty of Science, Toho University, 2-2-1 Miyama, 274-8510 Funabashi, Japan \\ e-mail: ozeki@env.sci.toho-u.ac.jp \\ 2 Department of Physics, Faculty of Science, University of Toyama, 3190 Gofuku, 930-8555 Toyama, Japan \\ e-mail: kaori@sci.u-toyama.ac.jp
}

Received 8 January 2014 / Accepted 23 April 2014

\begin{abstract}
Context. Methanimine $\left(\mathrm{CH}_{2} \mathrm{NH}\right)$ is a simple molecule composed of methylene and imine. The molecule has been detected toward the Galactic center, star-forming regions, circumstellar envelopes, and other galaxies since 1973. In previous studies, the rest frequency of methanimine has been measured for normal species up to the 650-GHz region, but its ${ }^{13} \mathrm{CH}_{2} \mathrm{NH}, \mathrm{CH}_{2}{ }^{15} \mathrm{NH}$, and $\mathrm{CH}_{2} \mathrm{ND}_{\text {isotopologs }}$ were limited to the $100-\mathrm{GHz}$ region.

Aims. If a rotational temperature of $100 \mathrm{~K}$ is assumed for methanimine, the highest intensity falls at approximately $1.5 \mathrm{THz}$. In addition to normal methanimine, the ${ }^{13} \mathrm{CH}_{2} \mathrm{NH}, \mathrm{CH}_{2}{ }^{15} \mathrm{NH}$, and $\mathrm{CH}_{2} \mathrm{ND}$ isotopologs in their ground-vibrational states were observed in the frequency range of $120-1600 \mathrm{GHz}$ to provide accurate rest frequency information. Based on this study, the calculated rest frequencies below $2 \mathrm{THz}$ should be sufficiently precise and support observations using all ALMA and Herschel/HIFI observational bands.

Methods. Methanimine was generated by pyrolysis of diaminoethane (DAE) vapor at $850{ }^{\circ} \mathrm{C}^{13} \mathrm{CH}_{2} \mathrm{NH}$ and $\mathrm{CH}_{2}{ }^{15} \mathrm{NH}_{\text {isotopologs }}$ were measured with their natural abundance, and deuterization of DAE was performed by mixing normal DAE with deuterated water, $\mathrm{D}_{2} \mathrm{O}$, and then pyrolyzed. This gives the deuterated isotopolog of methanimine, $\mathrm{CH}_{2} \mathrm{ND}$. Spectral measurements were performed by using the $23 \mathrm{kHz}$ source-frequency modulated terahertz spectrometer at Toho University.

Results. Both $a$ - and $b$-type transitions up to $1.6 \mathrm{THz}$ for the three isotopologs and the normal species were measured. Rotational and centrifugal distortion constants for the three isotopologs were accurately determined. For normal species $\left(\mathrm{CH}_{2} \mathrm{NH}\right)$, both electric quadrupole and nuclear spin-rotation coupling constants for nitrogen nucleus were determined, while for the ${ }^{13} \mathrm{CH}_{2} \mathrm{NH}$ and $\mathrm{CH}_{2} \mathrm{ND}$ species, only electric quadrupole-coupling constants for nitrogen nucleus were determined.

Conclusions. Our spectral line frequencies are suitable for a future astronomical search for these isotopologs of methanimine. The $1 \sigma$ frequency accuracy up to $2 \mathrm{THz}$ is lower than $100 \mathrm{kHz}$.
\end{abstract}

Key words. line: identification - molecular data - ISM: molecules - submillimeter: ISM

\section{Introduction}

In our previous paper (Motoki et al. 2013), we have reported terahertz spectroscopy of aminoacetonitrile $\left(\mathrm{NH}_{2} \mathrm{CH}_{2} \mathrm{CN}\right)$ and its amino-deuterated isotopologs. Aminoacetonitrile is one of the key molecules in Strecker-type synthesis, which was originally proposed in the laboratory condition (Strecker 1850) to form glycine, the simplest amino acid. This synthesis procedure starts with ammonia $\left(\mathrm{NH}_{3}\right)$ and formaldehyde $\left(\mathrm{H}_{2} \mathrm{CO}\right)$, which are well-known abundant species in interstellar space (for example, Cheung et al. 1968; Snyder et al. 1969):

$$
\begin{aligned}
& \mathrm{NH}_{3}+\mathrm{H}_{2} \mathrm{CO} \longrightarrow \mathrm{CH}_{2} \mathrm{NH}+\mathrm{H}_{2} \mathrm{O}, \\
& \mathrm{CH}_{2} \mathrm{NH}+\mathrm{HCN} \longrightarrow \mathrm{NH}_{2} \mathrm{CH}_{2} \mathrm{CN}, \\
& \mathrm{NH}_{2} \mathrm{CH}_{2} \mathrm{CN}+2 \mathrm{H}_{2} \mathrm{O} \longrightarrow \mathrm{NH}_{2} \mathrm{CH}_{2} \mathrm{COOH} \text { (glycine) }+\mathrm{NH}_{3} .
\end{aligned}
$$

To the best of our knowledge, glycine is the only one among the species appearing in the above series of reactions that has not yet been confirmed in interstellar space (Cheung et al. 1969; Buhl \& Snyder 1971). The detection of glycine was reported by Kuan et al. (2003), but it was not confirmed in a subsequent study (Snyder et al. 2005). For example, aminoacetonitrile, the direct precursor to glycine, was recently confirmed toward Sgr B2(N)

\footnotetext{
* The full line list is only available at the CDS via anonymous ftp to cdsarc.u-strasbg.fr (130.79.128.5) or via

http://cdsarc.u-strasbg.fr/viz-bin/qcat?J/A+A/566/A28
}

with a derived abundance on the order of $10^{16}$ molecules $\mathrm{cm}^{-2}$ (Belloche et al. 2008a,b).

In the reaction scheme of Strecker-type synthesis, methanimine $\left(\mathrm{CH}_{2} \mathrm{NH}\right)$ is a precursor of aminoacetonitrile. Because spectroscopic information on methanimine has been available since the early 1970 s and the molecule has a very simple structure consisting of methylene and imine groups, there have been numerous efforts to detect this molecule in space. On the basis of spectroscopic information, evidently, Godfrey et al. (1973) found interstellar methanimine for the first time toward the Galactic center in Sgr B2, measuring a column density of $3 \times 10^{14}$ molecules $\mathrm{cm}^{-2}$. Later, this molecule was also identified in several interstellar environments, such as hot cores associated with massive star-forming regions (for example, Qin et al. 2010), translucent molecular clouds (Turner et al. 1999), carbonrich circumstellar envelopes (for example, Tenenbaum et al. 2010), and extragalactic environments (for example, Martin et al. 2006). Although spectroscopy of the molecule in the 1970s was limited up to the millimeter-wave region (below $100 \mathrm{GHz}$ ), Dore et al. (2012) recently provided the rest frequency of this molecule by measuring the spectrum in the frequency range of 329-629 GHz. This enables us to search for this molecule with a frequency uncertainty of several kilohertz, or a radial velocity of lower than $0.01 \mathrm{~km} \mathrm{~s}^{-1}$ up to $1 \mathrm{THz}$. However, as shown in the figure in the paper by Dore et al. (2012), the spectral intensity of this molecule has its maximum in the submillimeter-wave region 


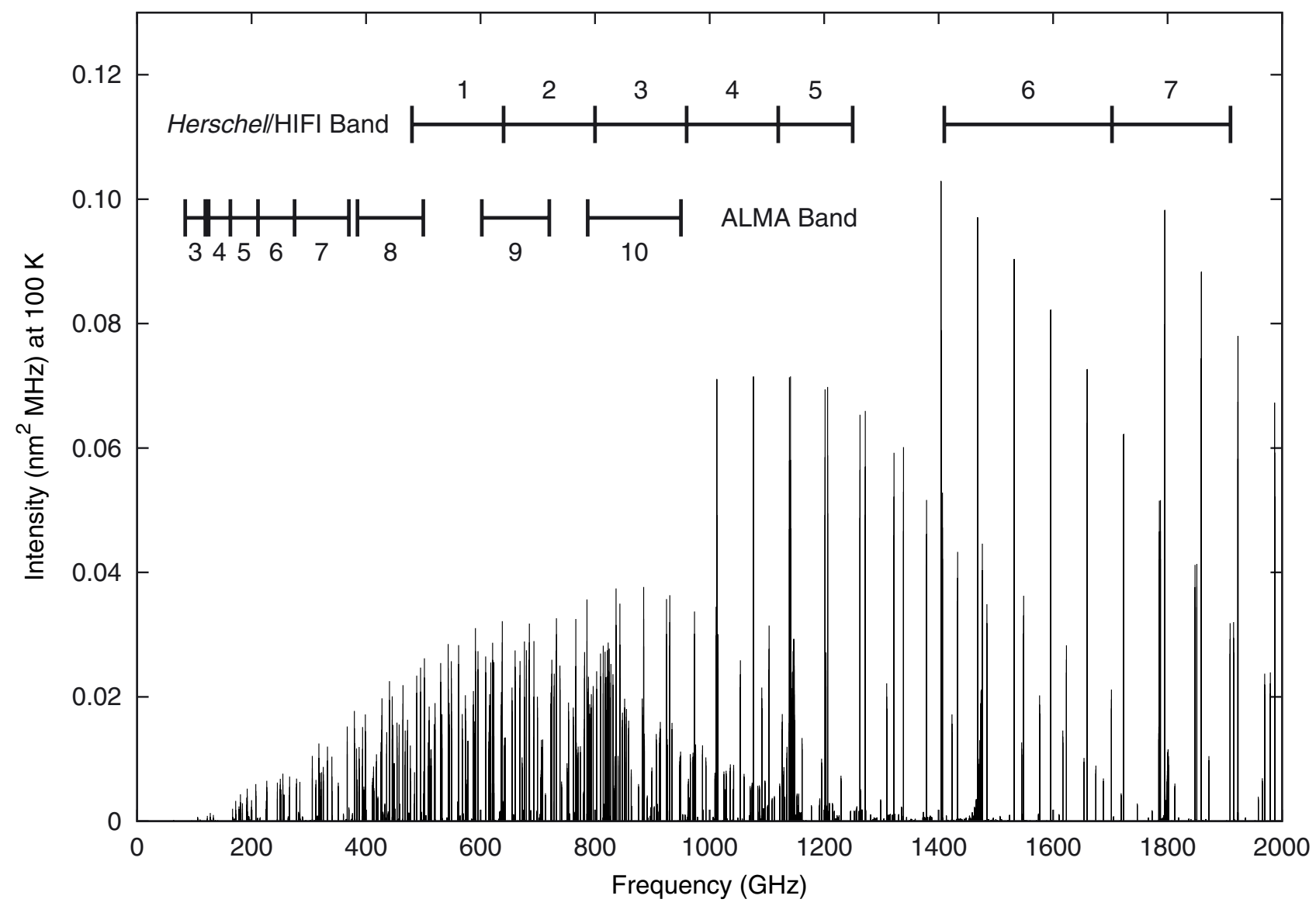

Fig. 1. Spectral intensity distribution of $\mathrm{CH}_{2} \mathrm{NH}$ at $100 \mathrm{~K}$ Both $a$-type and $b$-type transitions are depicted. The ALMA and Herschel band designations are also shown. As described in the text, the most intense peak appeared around $1.5 \mathrm{THz}$, which is a common feature for all other isotopologs studied in the present paper.

at rotational temperature conditions of $50-100 \mathrm{~K}$. The calculated spectral intensity of $\mathrm{CH}_{2} \mathrm{NH}$ at $100 \mathrm{~K}$ up to $2 \mathrm{THz}$ is shown in Fig. 1. The band designations of ALMA and Herschel/HIFI are also shown in the figure. The most intense lines lie at around $1.5 \mathrm{THz}$ and are stronger by three orders of magnitude than those in the millimeter-wave region. The molecule $\mathrm{CH}_{2} \mathrm{NH}$ was already identified in the millimeter-wave region and it is strongly expected to be observed in the submillimeterwave to terahertz-frequency region. The isotopologs ${ }^{13} \mathrm{CH}_{2} \mathrm{NH}$, $\mathrm{CH}_{2}{ }^{15} \mathrm{NH}$, and $\mathrm{CH}_{2} \mathrm{ND}$ may be detectable in the higher frequency region. Relevant spectroscopic information will be indispensable for their detection. Although the methylene-hydrogen deuterated methanimine can be considered as the singly substituted isotopologs, we have not tried to measure these species (two kinds of CHDNH) primarily because of the difficulty in the sample preparation. We therefore focused on the three isotologs in the present work.

Methanimine spectroscopy was performed for the first time by Johnson \& Lovas (1972). They measured the normal species of methanimine, $\mathrm{CH}_{2} \mathrm{NH}$, in the frequency region of $60-120 \mathrm{GHz}$ and determined rotational constants and quadrupole coupling constants of the nitrogen nucleus. Later, the measurement was extended by several investigators and were summarized by Kirchhoff et al. (1973), and parts of the lines were remeasured precisely by Krause et al. (1989). Pearson \& Lovas (1977) have been measuring various isotopologs of methanimine including ${ }^{13} \mathrm{CH}_{2} \mathrm{NH}, \mathrm{CH}_{2}{ }^{15} \mathrm{NH}$, and three kinds of deuterium isotopologs $\left(\mathrm{CD}_{2} \mathrm{ND}, \mathrm{CD}_{2} \mathrm{NH}\right.$, and $\left.\mathrm{CH}_{2} \mathrm{ND}\right)$ in the millimeter-wave region to determine their molecular structure.
Very recently, two spectroscopic studies have been reported by Dore and his collaborators (Dore et al. 2010, 2012), as mentioned before. The former study describes the determination of very precise hyperfine structure of $\mathrm{CH}_{2} \mathrm{NH}$ by using the Lambdip technique in the millimeter-wave region, by which not only electric quadrupole coupling constants of the nitrogen nucleus, but also the nuclear spin-rotation and nuclear spin-nuclear spin coupling constants of both $\mathrm{N}$ and $\mathrm{H}$ nuclei have been precisely determined. The latter study was mainly devoted to providing accurate rest frequencies of $\mathrm{CH}_{2} \mathrm{NH}$ for astronomers in the submillimeter-wavelength region. The rest frequencies provided were claimed to be sufficiently accurate for whole ALMA observational bands (with $1 \sigma$ uncertainties lower than $0.01 \mathrm{~km} \mathrm{~s}^{-1}$ in equivalent radial velocity). By combining their data with their analysis, Dore et al. $(2010,2012)$ provided not only extremely precise data in the millimeter-wave region, but also relatively higher frequency data.

We report in this paper terahertz spectroscopy of the three isotopologs $\left({ }^{13} \mathrm{CH}_{2} \mathrm{NH}, \mathrm{CH}_{2}{ }^{15} \mathrm{NH}\right.$, and $\left.\mathrm{CH}_{2} \mathrm{ND}\right)$ as well as main species. The measurements have been taken up to $1.6 \mathrm{THz}$ to provide accurate rest frequencies up to at least $2 \mathrm{THz}$, covering all ALMA and Herschel/HIFI observational bands and with sufficient accuracy of $<0.1 \mathrm{MHz}$, corresponding to radial velocities of $<0.02 \mathrm{~km} \mathrm{~s}^{-1}$.

\section{Experiment}

Methanimine was generated by pyrolysis of the alkylamine diaminoethane $\left(\mathrm{NH}_{2} \mathrm{CH}_{2} \mathrm{CH}_{2} \mathrm{NH}_{2}\right)$ in the present study. A quartz 
Y. Motoki et al.: Terahertz spectroscopy of methanimine and its isotopologs

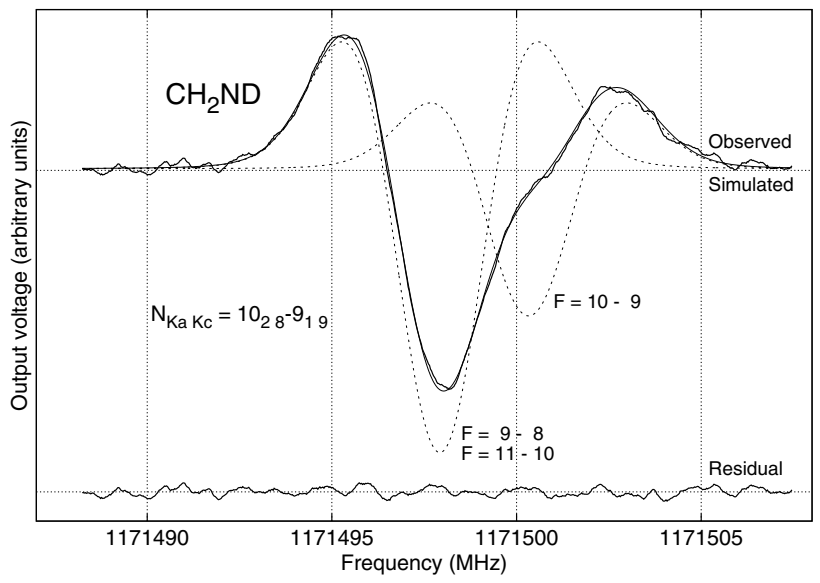

Fig. 2. Observed spectra (solid line) of $\mathrm{CH}_{2} \mathrm{ND}\left(N_{K_{a} K_{c}}=10_{28} \leftarrow 9_{19}\right)$. The nitrogen hyperfine structure (quadrupole coupling) was partially resolved. They are completely decomposed into two spectral components (overlapping of $F=11-10$ and 9-8 and $F=10-9$ components) shown as dotted lines, each of which has been simulated by giving appropriate parameters to make the second derivative of Voigt line shapes. The residual between observed and simulated spectra was satisfactorily small, as shown at the bottom of the figure. The spectrum was recorded by integrating 30 scans with a $0.5 \mathrm{~Hz}$ repetition rate and a $1 \mathrm{~ms}$ time constant of the lock-in amplifier.

tube of $1 / 4$ inch in diameter and $50 \mathrm{~cm}$ in length was heated in a furnace over a length of approximately $30 \mathrm{~cm}$. The pyrolysis temperature was optimized to $850^{\circ} \mathrm{C}$ by monitoring the intensity of known spectral lines of the normal species in the millimeterwave region (Dore et al. 2010). Diaminoethane was continuously flowed through the heated quartz tube and the pyrolysis product was directly introduced into the absorption cell. No other buffer gas was introduced into the absorption cell. The pressure of the cell was kept at approximately 2-4 Pa. Observation of ${ }^{13} \mathrm{CH}_{2} \mathrm{NH}$ and $\mathrm{CH}_{2}{ }^{15} \mathrm{NH}$ was made in natural abundances. The imino-hydrogen deuterated methanimine $\left(\mathrm{CH}_{2} \mathrm{ND}\right)$ was from the deuterium-enriched precursor, which was prepared by mixing deuterated water with diaminoethane in a volume ratio of 1:2. All spectral lines were measured with the $23 \mathrm{kHz}$-frequency modulated terahertz spectrometer at Toho University. The details of our spectrometer have been described extensively in our previous study (Motoki et al. 2013). Millimeter-wave to terahertz radiation in the frequency range of $120-1600 \mathrm{GHz}$ was provided by the state-of-the-art frequency multiplier chains (VDI Inc.) output from a microwave synthesizer (Agilent 8257D). Both a GaAs solid-state zero-bias detector that operates at room temperature (VDI Inc.) and a liquid-helium-cooled InSb bolometer (QMC Instruments Ltd.) were used. The former was used mainly for detection below $1 \mathrm{THz}$ and the latter above $1 \mathrm{THz}$. The 40-60 dB preamplified signal is detected at twice the modulation frequency, giving a second-derivative line shape. An example of the measured spectrum is shown in Fig. 2 to show the signal-to-noise raio of $\mathrm{CH}_{2} \mathrm{ND}$. Because the spectra of ${ }^{13} \mathrm{CH}_{2} \mathrm{NH}$ and $\mathrm{CH}_{2}{ }^{15} \mathrm{NH}$ were recorded in natural abundances, numerous scans were necessary to obtain the same signal-to-noise ratio as that for the normal species. In Table 1, a part of the observed frequencies at around $1.5 \mathrm{THz}$ is listed. The frequency measurement error in this frequency region is approximately $70 \mathrm{kHz}$. We estimated the error of the measured frequency in the present study to be between 30 and $70 \mathrm{kHz}$ depending on the observed frequencies. Of course, the given frequency error becomes smaller as the measurement frequency becomes lower.
Table 1. Selection of observed transition frequencies of $\mathrm{CH}_{2} \mathrm{NH}$ and its isotopologs around $1500 \mathrm{GHz}$.

\begin{tabular}{|c|c|c|c|}
\hline Species & $J_{K_{a^{\prime}} K_{c^{\prime}}}^{\prime} \leftarrow J_{K_{a} K_{c}}$ & Frequency $^{a}$ & obs. - calc. \\
\hline \multirow{13}{*}{$\mathrm{CH}_{2} \mathrm{NH}$} & $4_{40} \leftarrow 3_{31}$ & $1404238.028(70)^{b}$ & 0.140 \\
\hline & $5_{42} \leftarrow 4_{31}$ & $1468185.402(70)$ & -0.007 \\
\hline & $5_{41} \leftarrow 4_{32}$ & $1468199.075(70)$ & -0.023 \\
\hline & $12_{57} \leftarrow 12_{48}$ & $1471199.202(70)$ & -0.012 \\
\hline & $12_{58} \leftarrow 12_{49}$ & $1471499.403(70)$ & -0.027 \\
\hline & $9_{54} \leftarrow 9_{45}$ & $1473861.534(70)$ & -0.018 \\
\hline & $9_{55} \leftarrow 9_{46}$ & $1473892.068(70)$ & -0.014 \\
\hline & $8_{53} \leftarrow 8_{44}$ & $1474356.250(70)$ & 0.024 \\
\hline & $8_{54} \leftarrow 8_{45}$ & $1474367.926(70)$ & -0.089 \\
\hline & $11_{39} \leftarrow 10_{28}$ & $1484492.817(70)$ & -0.011 \\
\hline & $12_{310} \leftarrow 11_{29}$ & $1532209.417(70)$ & -0.076 \\
\hline & $11_{38} \leftarrow 10_{29}$ & $1548586.974(70)$ & 0.002 \\
\hline & $13_{311} \leftarrow 12_{210}$ & $1576363.188(70)$ & -0.010 \\
\hline \multirow{19}{*}{${ }^{13} \mathrm{CH}_{2} \mathrm{NH}$} & $21_{219} \leftarrow 20_{218}$ & $1352287.798(70)$ & 0.012 \\
\hline & $22_{121} \leftarrow 21_{120}$ & $1357924.664(70)$ & -0.046 \\
\hline & $4_{41} \leftarrow 3_{30}$ & $1403358.349(70)$ & 0.052 \\
\hline & $4_{40} \leftarrow 3_{31}$ & $1403359.871(70)$ & -0.096 \\
\hline & $11_{39} \leftarrow 10_{28}$ & $1474883.686(70)$ & -0.022 \\
\hline & $14_{59} \leftarrow 14_{410}$ & $1475381.288(70)$ & 0.058 \\
\hline & $14_{510} \leftarrow 14_{411}$ & $1476200.023(70)$ & -0.039 \\
\hline & $13_{59} \leftarrow 13_{410}$ & $1477506.681(70)$ & -0.015 \\
\hline & $12_{57} \leftarrow 12_{48}$ & $1478359.598(70)$ & 0.083 \\
\hline & $11_{56} \leftarrow 11_{47}$ & $1479383.508(70)$ & 0.023 \\
\hline & $11_{57} \leftarrow 11_{48}$ & $1479506.536(70)$ & -0.022 \\
\hline & $10_{55} \leftarrow 10_{46}$ & $1480174.884(70)$ & -0.010 \\
\hline & $10_{56} \leftarrow 10_{47}$ & $1480232.612(70)$ & -0.001 \\
\hline & $9_{54} \leftarrow 9_{45}$ & $1480778.809(70)$ & 0.070 \\
\hline & $9_{55} \leftarrow 9_{46}$ & $1480803.406(70)$ & -0.177 \\
\hline & $12_{310} \leftarrow 11_{29}$ & $1522368.746(70)$ & -0.126 \\
\hline & $6_{43} \leftarrow 5_{32}$ & $1528120.333(70)$ & -0.016 \\
\hline & $6_{42} \leftarrow 5_{33}$ & $1528167.416(70)$ & 0.024 \\
\hline & $13_{311} \leftarrow 12_{210}$ & $1566486.726(70)$ & 0.011 \\
\hline \multirow[t]{22}{*}{$\mathrm{CH}_{2}{ }^{15} \mathrm{NH}$} & $22_{320} \leftarrow 21_{319}$ & $1372963.250(70)$ & -0.039 \\
\hline & $22_{617} \leftarrow 21_{616}$ & $1376401.678(70)$ & 0.044 \\
\hline & $22_{616} \leftarrow 21_{615}$ & $1376428.341(70)$ & 0.061 \\
\hline & $22_{518} \leftarrow 21_{617}$ & $1379173.653(70)$ & -0.043 \\
\hline & $22_{517} \leftarrow 21_{616}$ & $1379791.274(70)$ & -0.082 \\
\hline & $22_{418} \leftarrow 21_{417}$ & $1389424.015(70)$ & 0.017 \\
\hline & $21_{420} \leftarrow 20_{119}$ & $1392881.468(70)$ & -0.081 \\
\hline & $24_{024} \leftarrow 23_{123}$ & $1401369.234(70)$ & -0.042 \\
\hline & $24_{124} \leftarrow 23_{123}$ & $1403565.546(70)$ & -0.034 \\
\hline & $24_{024} \leftarrow 23_{023}$ & $1404280.946(70)$ & 0.010 \\
\hline & $24_{123} \leftarrow 23_{222}$ & $1407122.642(70)$ & -0.042 \\
\hline & $23_{122} \leftarrow 22_{122}$ & $1413143.255(70)$ & -0.037 \\
\hline & $22_{518} \leftarrow 22_{419}$ & $1455861.400(70)$ & -0.046 \\
\hline & $24_{223} \leftarrow 23_{222}$ & $1457357.254(70)$ & 0.000 \\
\hline & $25_{025} \leftarrow 24_{124}$ & $1459068.714(70)$ & 0.032 \\
\hline & $7_{52} \leftarrow 7_{43}$ & $1477594.406(70)$ & 0.073 \\
\hline & $7_{53} \leftarrow 7_{44}$ & $1477597.522(70)$ & -0.060 \\
\hline & $5_{5} \leftarrow 5_{4}$ & $1478001.450(70)$ & -0.064 \\
\hline & $24_{421} \leftarrow 23_{420}$ & $1507131.016(70)$ & 0.009 \\
\hline & $25_{224} \leftarrow 24_{223}$ & $1515322.641(70)$ & 0.024 \\
\hline & $24_{223} \leftarrow 23_{122}$ & $1519421.807(70)$ & 0.061 \\
\hline & $24_{420} \leftarrow 23_{419}$ & $1520980.865(70)$ & 0.007 \\
\hline \multirow[t]{11}{*}{$\mathrm{CH}_{2} \mathrm{ND}$} & $8_{45} \leftarrow 7_{34}$ & $1365724.764(70)$ & 0.002 \\
\hline & $8_{44} \leftarrow 7_{35}$ & $1366494.752(70)$ & 0.014 \\
\hline & $9_{46} \leftarrow 8_{35}$ & $1423367.321(70)$ & 0.009 \\
\hline & $9_{45} \leftarrow 8_{36}$ & $1425064.589(70)$ & 0.012 \\
\hline & $5_{5} \leftarrow 4_{4}$ & $1446408.397(70)$ & 0.077 \\
\hline & $10_{47} \leftarrow 9_{36}$ & $1480328.684(70)$ & 0.017 \\
\hline & $10_{46} \leftarrow 9_{37}$ & $1483724.746(70)$ & 0.009 \\
\hline & $11_{48} \leftarrow 10_{37}$ & $1536302.549(70)$ & 0.000 \\
\hline & $11_{47} \leftarrow 10_{38}$ & $1542599.447(70)$ & 0.012 \\
\hline & $7_{53} \leftarrow 6_{42}$ & $1563606.214(70)$ & -0.031 \\
\hline & $7_{52} \leftarrow 6_{43}$ & $1563609.067(70)$ & 0.105 \\
\hline
\end{tabular}

Notes. Hyperfine splitting due to the nitrogen nucleus is not resolved for the tabulated lines. See text for the detail. (a) Units are in MHz. (b) Experimental frequency accuracy in the present study. 
Table 2. Molecular constants of methanimine and its isotopologs.

\begin{tabular}{|c|c|c|c|c|}
\hline Parameter & $\mathrm{CH}_{2} \mathrm{NH}$ & ${ }^{13} \mathrm{CH}_{2} \mathrm{NH}$ & $\mathrm{CH}_{2}{ }^{15} \mathrm{NH}$ & $\mathrm{CH}_{2} \mathrm{ND}$ \\
\hline$A(\mathrm{MHz})$ & $196210.87629(49)^{a}$ & $196195.4866(44)$ & $195737.5201(43)$ & $157673.92406(169)$ \\
\hline$B(\mathrm{MHz})$ & $34641.699423(87)$ & $33747.23050(117)$ & $33735.38602(79)$ & $32069.00792(41)$ \\
\hline$C(\mathrm{MHz})$ & $29351.496787(81)$ & $28706.32768(110)$ & $28687.85279(65)$ & $26563.45064(44)$ \\
\hline$D_{J}(\mathrm{kHz})$ & $56.00411(44)$ & $53.4737(49)$ & $53.43278(237)$ & $49.92710(163)$ \\
\hline$D_{J K}(\mathrm{kHz})$ & $598.6775(54)$ & $585.589(39)$ & $580.029(34)$ & $327.4730(110)$ \\
\hline$D_{K}(\mathrm{kHz})$ & $6383.483(36)$ & 6393.40(35) & 6356.61(43) & 4998.306(74) \\
\hline$d_{1}(\mathrm{kHz})$ & $-9.973179(80)$ & $-9.24134(313)$ & $-9.26331(162)$ & $-10.90309(63)$ \\
\hline$d_{2}(\mathrm{kHz})$ & $-2.007905(34)$ & $-1.83540(104)$ & $-1.83380(52)$ & $-2.069013(219)$ \\
\hline$H_{J}(\mathrm{~Hz})$ & $0.01736(48)$ & $0.0208(69)$ & $0.01895(253)$ & $0.04504(186)$ \\
\hline$H_{J K}(\mathrm{~Hz})$ & $2.6068(112)$ & $2.445(77)$ & $2.370(41)$ & $1.9751(232)$ \\
\hline$H_{K J}(\mathrm{~Hz})$ & $6.125(69)$ & $7.38(74)$ & $7.69(78)$ & $-32.070(180)$ \\
\hline$H_{K}(\mathrm{~Hz})$ & $877.75(104)$ & $861.8(90)$ & $876.6(117)$ & $779.52(147)$ \\
\hline$h_{1}(\mathrm{mHz})$ & $33.067(164)$ & $26.7(53)$ & $30.35(200)$ & $43.08(92)$ \\
\hline$h_{2}(\mathrm{mHz})$ & $32.462(61)$ & $23.9(34)$ & $25.85(96)$ & $36.91(60)$ \\
\hline$h_{3}(\mathrm{mHz})$ & $9.091(39)$ & $7.38(190)$ & $9.11(96)$ & $10.013(224)$ \\
\hline$L_{K}(\mathrm{mHz})$ & $-163.0(47)$ & & & \\
\hline$l_{1}(\mu \mathrm{Hz})$ & $-0.541(97)$ & & & \\
\hline$l_{3}(\mu \mathrm{Hz})$ & $-0.472(34)$ & & & \\
\hline$\chi_{a a}(\mathrm{~N})(\mathrm{MHz})$ & $-0.91523(141)$ & $-0.568(109)$ & & $-0.5268(316)$ \\
\hline$\chi_{b b}(\mathrm{~N})(\mathrm{MHz})$ & $-2.66739(126)$ & $-2.881(71)$ & & $-3.0186(209)$ \\
\hline$C_{a a}(\mathrm{~N})(\mathrm{kHz})$ & $53.16(71)$ & $53.2^{b}$ & & $42.8^{b}$ \\
\hline$C_{b b}(\mathrm{~N})(\mathrm{kHz})$ & $9.351(230)$ & $9.09^{b}$ & & $8.64^{b}$ \\
\hline$C_{c c}(\mathrm{~N})(\mathrm{kHz})$ & $1.214(215)$ & $1.17^{b}$ & & $1.09^{b}$ \\
\hline \# of newly measured lines & 201 & 143 & 136 & 239 \\
\hline$\#$ of fitted lines ${ }^{c}$ & $389^{d}$ & $146^{e}$ & $139^{e}$ & $268^{e}$ \\
\hline $\mathrm{rms}$ & 0.869 & 0.908 & 0.750 & 0.619 \\
\hline
\end{tabular}

Notes. ${ }^{(a)}$ Values in parentheses denote one standard deviation and apply to the last digits of the constants. ${ }^{(b)}$ Fixed. See text in detail. ${ }^{(c)}$ In addition to the newly observed lines in the present study, available data have been included for the analysis. ${ }^{(d)}$ Data observed by Johnson \& Lovas (1972); Kirchhoff et al. (1973); Krause et al. (1989); Dore et al. (2010); and Dore et al. (2012) are also included in the analysis. ${ }^{(e)}$ Data observed by Pearson \& Lovas (1977) are also included in the analysis.

For the lines showing asymmetric line shape, we tried to obtain each frequency by decomposing each hyperfine component frequency as shown in Fig. 2. When hyperfine components were not resolved, the frequency of the overlapping line is the intensity-weighted average of the corresponding components. The expected intensity of normal methanimine is so high (as can be seen in Fig. 1) that we may observe the distorted spectral line shape due to optical thickness for the intense line over $1 \mathrm{THz}$. However, we did not observe this effect because we were able to fit the spectral profile normally with a simple Voigt function for all the observed lines, probably because our pyrolysis condition was not efficient and the optically thin condition was virtually realized.

\section{Spectral analysis}

$\mathrm{CH}_{2} \mathrm{NH}$ is a nearly prolate rotor $(\kappa=-0.937)$ and the $\mathrm{CN}$ molecular axis lies almost along the $a$ axis. The dipole moment of the molecule is mainly governed by $\mathrm{CN}$ and $\mathrm{NH}$ groups, with a CNH angle of approximately $110^{\circ}$ (Pearson \& Lovas 1977). This means that the dipole moment lies in the middle of the $a$ and $b$ axes. The dipole moment of the normal species was measured by Allegrini et al. (1978) by laser Stark spectroscopy, giving quite similar components for both $a$ and $b$ axes $\left(\mu_{a}\right.$ and $\mu_{b}$ of 1.3396(30) and 1.4461(90) Debyes, respectively). From the view point of astronomical observation, both types of transitions are important.

The observed spectral-line frequency data were analyzed using the following S-reduced Hamiltonian for an asymmetric top molecule:

$H=H_{\mathrm{rot}}+H_{\mathrm{eQq}, \mathrm{N}}+H_{\mathrm{nsr}, \mathrm{N}}$ where $H_{\text {rot }}$ represents the rotational Hamiltonian including the centrifugal distortion effect. For the analysis of the normal species, three octic centrifugal terms were needed because we included higher $N$ and $K_{a}$ lines in the fitting. This choice of molecular parameter set is coherent with the data set archived in the Cologne Database for Microwave Spectroscopy (CDMS; Müller et al. 2001, 2005). For other isotopologs, sextic centrifugal distortion constants are sufficient to explain all the observed line frequencies. $H_{\mathrm{eQq}, \mathrm{N}}$ and $H_{\mathrm{nsr}, \mathrm{N}}$ denote the Hamiltonians for an electric quadrupole interaction and nuclear spin-rotation interaction for the nitrogen nucleus, respectively. The former was taken for all species with nitrogen-14 nuclei; for the latter interaction term, however, it was included only for the normal species because we also fitted the line frequency observed previously by Dore et al. (2010) using the Lamb-dip technique in the millimeter-wave region. In the present analysis, we only considered the nitrogen hyperfine coupling described above because we were not able to observe any spectral line splitting from nuclei other than nitrogen. Thus, the coupling scheme we employed in the present analysis is

$N+I(N)=F \quad$ with $\quad I(N)=1$.

The observed data were combined with the data obtained previously (Johnson \& Lovas 1972; Kirchhoff et al. 1973; Pearson \& Lovas 1977; Krause et al. 1989; Dore et al. 2010, 2012) and were analyzed by using the SPFIT/SPCAT suite of programs (Pickett 1991). The number of measured lines in the present study and the fitted lines as well as the determined molecular constants for all four isotopologs are summarized in Table 2. Among hyperfine coupling constants, nitrogen nuclear spin-rotation coupling constants for the ${ }^{13} \mathrm{CH}_{2} \mathrm{NH}$ and $\mathrm{CH}_{2} \mathrm{ND}$ isotopologs were poorly 
Table 3. Partition function of methanimine and its isotopomers.

\begin{tabular}{ccccc}
\hline \hline Temperature (K) & $\mathrm{CH}_{2} \mathrm{NH}$ & ${ }^{13} \mathrm{CH}_{2} \mathrm{NH}$ & $\mathrm{CH}_{2}{ }^{15} \mathrm{NH}$ & $\mathrm{CH}_{2} \mathrm{ND}$ \\
\hline 300 & 5903.9 & 6048.5 & 2019.3 & 7195.1 \\
225 & 3834.9 & 3928.7 & 1311.8 & 4673.2 \\
150 & 2088.6 & 2139.7 & 714.43 & 2544.9 \\
100 & 1138.34 & 1166.15 & 389.36 & 1386.78 \\
75 & 740.42 & 758.48 & 253.25 & 901.87 \\
37.5 & 263.37 & 269.76 & 90.069 & 320.60 \\
18.75 & 94.295 & 96.554 & 32.238 & 114.64 \\
9.375 & 34.207 & 35.007 & 11.688 & 41.482 \\
\hline
\end{tabular}

Notes. Tabulated are spin-rotation partition functions, for which only ${ }^{14} \mathrm{~N}$ spin is considered.

determined mainly because of paucity of observed hyperfine resolved spectral lines. These constants were estimated from corresponding constants for normal species by scaling the ratio of rotational constants, and were included as the fixed constants in the present analysis. We will deposit the line lists in the CDMS ${ }^{1}$, the Toyama Microwave Atlas (ToyaMA) ${ }^{2}$, and Strasbourg astronomical Data Center $(\mathrm{CDS})^{3}$ for the creation of new or/and updated entries.

\section{Results and discussion}

We measured pure rotational spectra of methaneimine and its isotopologs ${ }^{13} \mathrm{CH}_{2} \mathrm{NH}, \mathrm{CH}_{2}{ }^{15} \mathrm{NH}$, and $\mathrm{CH}_{2} \mathrm{ND}$ up to $1.6 \mathrm{THz}$. This enabled us to predict astronomically important rotational line frequencies of these species with accuracies of $<100 \mathrm{kHz}$ even at $1.91 \mathrm{THz}$, the upper frequency limit of Herschel/HIFI band 7 . This error corresponds to a radial velocity of $15 \mathrm{~m} \mathrm{~s}^{-1}$. The frequency accuracy becomes better with lower frequency. For example, at $1 \mathrm{THz}$, the typical frequency error for the line whose upper state energy is below $100 \mathrm{~cm}^{-1}$ for $\mathrm{CH}_{2} \mathrm{ND}$ is $\lesssim 10 \mathrm{kHz}$, corresponding to a radial velocity of $3 \mathrm{~m} \mathrm{~s}^{-1}$.

The rotational partition functions for several temperatures were calculated and are listed in Table 3 . It is worth mentioning that the intensity of the spectra for all isotopologs was estimated by using the estimated values of electric dipole moments for three isotopologs from the corresponding values of $\mathrm{CH}_{2} \mathrm{NH}$, which are experimentally determined. Upon exchanging an isotopic atom, the molecular axis should rotate by several degrees, while the direction of the electric dipole moment should be essentially unchanged. The projection onto each molecular axis is thus changed in accordance with the rotation angle of the $a-b$ molecular axes. This effect is most prominent in the case of deuterated metahimine, $\mathrm{CH}_{2} \mathrm{ND}$, as the molecular axis will rotate by several degrees with respect to the normal species within an $a-b$ molecular axis plane. This rotation of the molecular axis results in a change of the electric dipole moment components for $\mathrm{CH}_{2} \mathrm{ND}$, i.e., the estimated $\mu_{a}$ and $\mu_{b}$ are 1.23 and 1.54 Debyes, respectively. This leads to a change in the calculated intensity by approximately $20 \%$ compared with the values without this correction. As already mentioned above, since both carbon and nitrogen nuclei lie almost along the $a$ axis, the effect of substituting $\mathrm{N}$ or $\mathrm{C}$ atoms is weaker than substituting imino-hydrogen by a deuterium atom. This estimation of the spectral intensity is probably sufficiently accurate at a level

\footnotetext{
http://www . astro.uni-koeln.de/cdms

2 http://www.sci.u-toyama.ac.jp/phys/4ken/atlas/

3 http://cdsweb.u-strasbg.fr/
}

of $5 \%$. Other contributions to the uncertainties of the spectral intensity are the low-lying vibrationally excited state and truncation of rotational levels in calculating the partition function. For methanimine, most of the vibrational frequencies have been already measured by other authors (Allegrini et al. 1978; Duxbury et al. 1981; Hamada et al. 1984; Halonen \& Duxubury 1985). We found that all the reported vibrational frequencies are higher than $1000 \mathrm{~cm}^{-1}$; the effect on the spectral intensity from this factor is thus negligibly small under the conditions of relatively cold interstellar space of 10 to $100 \mathrm{~K}$, for example. The effect of truncation of rotational levels ( $J \leq 99$ in the present study) was also checked by making a comparison with the approximate formula (Gordy \& Cook 1984), and we confirmed that the effect on the spectral intensity is also negligibly small.

Finally we would like to mention the astronomical implication of this molecule. The normal species of methanimine has been observed toward several objects as described in the introduction section, to the best of our knowledge, however, no reports have been made with respect to the detection of the isotopologs, except for that of Cummins et al. (1986) who described the possible detection of ${ }^{13} \mathrm{C}$ isotopolog at $124 \mathrm{GHz}$ region toward Sgr B2. This assignment was based on the frequency coincidence of the single transition that has been measured in the laboratory (Pearson \& Lovas 1977). It is hard to confirm the detection of the molecules from this report alone. Another assignment of the transitions based on the present work will be necessary.

\section{Conclusions}

Spectral lines of methanimine and its three isotopologs $\left(\mathrm{CH}_{2} \mathrm{NH}\right.$, ${ }^{13} \mathrm{CH}_{2} \mathrm{NH}, \mathrm{CH}_{2}{ }^{15} \mathrm{NH}$, and $\mathrm{CH}_{2} \mathrm{ND}$ ) were observed in the laboratory up to $1600 \mathrm{GHz}$. The obtained frequency data were analyzed and the molecular constants as well as spin-rotational partition function at various temperatures were revised. The frequency error for spectral lines with upper-state energy below $100 \mathrm{~cm}^{-1}$ is $<100 \mathrm{kHz}$ even at $2 \mathrm{THz}$, corresponding to a radial velocity of $15 \mathrm{~m} \mathrm{~s}^{-1}$. The accuracy of the intensity is expected to be within 5\%. Our provided spectral line list will be cataloged in several spectral line databases and is valid for astronomical searches for methanimine in all ALMA and Herschel/HIFI observational bands.

Acknowledgements. H.O. thanks the Futaba Electronics Memorial Foundation for its financial support in constructing the spectrometer at Toho University. This study was supported by a Grant-in-Aid for Scientific Research (C) from the Ministry of Education, Culture, Sports, Science, and Technology of Japan (Grant No. 24540238).

\section{References}

Allegrini, M., Johns, J. W. C., \& McKellar, A. R. W. 1978, J. Chem. Phys., 70, 2829

Belloche, A., Menten, K. M., Comito, C., et al. 2008a, A\&A, 482, 179

Belloche, A., Menten, K. M., Comito, C., et al. 2008b, A\&A, 492, 769

Buhl, D., \& Snyder, L. E. 1971, ApJ, 163, L47

Cheung, A. C., Rank, D. M., Towns, C. H., Thornton, D. D., \& Welch, W. J. 1968, Phys. Rev. Lett., 21, 1701

Cheung, A. C., Rank, D. M., Towns, C. H., Thornton, D. D., \& Welch, W. J. 1969, Nature, 221, 626

Cummins, S. E., Linke, R. A., \& Thaddeus, P. 1986, ApJS, 60, 819

Dore, L., Bizzochi, L., Degli Esposti, C., \& Gauss, J. 2010, J. Mol. Spectrosc., 263, 44

Dore, L., Bizzochi, L., \& Degli Esposti, C. 2012, A\&A, 544, A19

Duxbury, G. Kato, H., \& Le Lerre, M. L. 1981, Discuss. Faraday Soc., 71, 97 
Godfrey, P. D., Brown, R. D., Robinson, B. J., \& Sinclair, W. M. 1973, Astrophys. Lett., 13, 119

Gordy, W., \& Cook, R. L. 1984, Microwave Molecular Spectra, 3rd edn (New York: Wiley)

Halonen, L., \& Duxubury, G. 1985, J. Chem. Phys., 83, 2078

Hamada, Y., Hashiguchi, K., Tsuboi, M. Koga, Y., \& Kondo, S. 1984, J. Mol. Spectrosc., 105, 70

Johnson, D. R., \& Lovas, F. J. 1972, Chem. Phys. Lett., 15, 65

Kirchhoff, W. H., Johnson, D. R., \& Lovas, F. J. 1973, J. Phys. Chem. Ref. Data, 2,1

Krause, H., Sutter, D. H. \& Palmer, M. H. 1989, Z. Naturaforsch. 44a, 1063

Kuan, Y.-J., Charnley, S. B., Huang, H.-C., Tseng, W.-L., \& Kisiel, Z. 2003, ApJ, 593, 848

Martin, S., Mauersberger, R., Martin-Pintado, J., Henkel, C., \& Garcia-Burillo, S. 2006, ApJ Suppl., 164, 450
Motoki, Y., Tsunoda, Y., Ozeki, H., \& Kobayashi, K. 2013, ApJS, 209, 23

Müller, H. S. P., Thornwirth, S., Roth, D. A., \& Winnewisser, G. 2001, A\&A, 370, L49

Müller, H. S. P., Schlöder, F., Stutzki, J., \& Winnewisser, G. 2005, J. Mol. Struct., 742,215

Pearson, Jr., R., \& Lovas, F. J. 1977, J. Chem. Phys., 66, 4149

Pickett, H. M. 1991, J. Mol. Spectrosc., 148, 371

Qin, S.-L., Wu, Y., Huang, M., et al. 2010, ApJ, 711, 399

Snyder, L. E., Buhl, D., Zuckerman, B., \& Palmer, P. 1969, Phys. Rev. Lett., 22, 679

Snyder, L. E., Lovas, F. J., Hollis, J. M., et al. 2005, ApJ, 619, 914

Strecker, A. 1850, Ann. Chem. Pharm., 75, 27

Tenenbaum, E. D., Dodd, J. L., Milam, S. N., Woolf, N. J., \& Ziurys, L. M. 2010, ApJ, 720, L102

Turner, B. E., Terzieva, R., \& Herbst, E. 1999, ApJ, 518, 699 